\title{
En este número de Endoscopia
}

\section{In this issue of Endoscopy}

Félix I. Téllez-Ávila*

Departamento de Endoscopia, Instituto Nacional de Ciencias Médicas y Nutrición Salvador Zubirán, Ciudad de México, México

En este número de Endoscopia nos encontramos de lleno en la pandemia de enfermedad por coronavirus 2019 en nuestro país. Iniciamos este editorial deseando que todos ustedes, así como sus seres queridos, se encuentren bien y juntos, y así podamos salir adelante de la situación mundial actual. Conscientes de la importancia de continuar con nuestras actividades académicas lo más cercano a normal compartimos con ustedes este número de la revista en el 2020.

En el presente número el grupo de endoscopia del Hospital Juárez de México nos muestra un interesante trabajo sobre los factores de riesgo asociados a una mala preparación colónica. Existen numerosos estudios de otros países donde se han reportado algunos de los factores en sus poblaciones, sin embargo, existe poca información proveniente de nuestro medio, por lo cual el trabajo actual cobra relevancia. Es bien sabido que la población de nuestro país tiene características particulares como la dieta, problemas de peso y comorbilidades principalmente de tipo metabólico. Todos estos factores fueron estudiados y considerados en el presente trabajo y consideramos importante su revisión por parte de los endoscopistas de nuestro medio.
El trabajo sobre heteropía gástrica en el esófago por el Dr. Hernández trata un tema poco estudiado en general y que puede llegar a plantear dudas sobre qué debemos hacer cuando encontramos un paciente con esta situación. Consideramos que el trabajo mencionado ayudará a los endoscopistas a tomar decisiones cuando tengan este hallazgo en sus pacientes.

Finalmente se presentan varios casos clínicos y trabajos de revisión interesantes en este número. Los trabajos sobre el papel de la endoscopia en el manejo de patologías pancreáticas comunes serán de relevancia para los interesados en los temas de patología pancreatobiliar y esperamos sean de utilidad y de su agrado.

Finalmente, deseamos de todo corazón que todos los miembros de la Asociación Mexicana de Endoscopia Gastrointestinal, médicos y no médicos, amigos y seres queridos nos mantengamos sanos y pasemos esta contingencia de la mejor manera posible. El Comité Editorial de Endoscopia les envía nuestros mejores deseos y todas nuestras oraciones.

Dr. Félix Ignacio Téllez-Ávila Editor en Jefe 